\title{
TORSION OF BEAMS OF L-CROSS-SECTION
}

\author{
by E. DEUTSCH
}

(Received 12 September, 1960; revised 3 September, 1961)

1. Introduction. The torsion of beams of L-cross-section was studied for the first time, from a mathematical standpoint, by Kötter [1]. He solved the problem in the case of an L-section both arms of which are infinite. Some time later, Trefftz [2], in his work on the torsion of beams of polygonal cross-section, applied his method also to an infinite L-section. In 1934, Seth [3] solved the case of a beam of an L-section with only one infinite arm. In 1949. Arutyanyan [4] solved the torsion problem of an L-section that has both arms finite, but of equal length, reducing the problem to that of solving an infinite system of equations.

In the present paper, we propose to solve the problem of the torsion of an L-section, in the general case of finite arms, not necessarily equal and not equally thick. See Fig. 1.

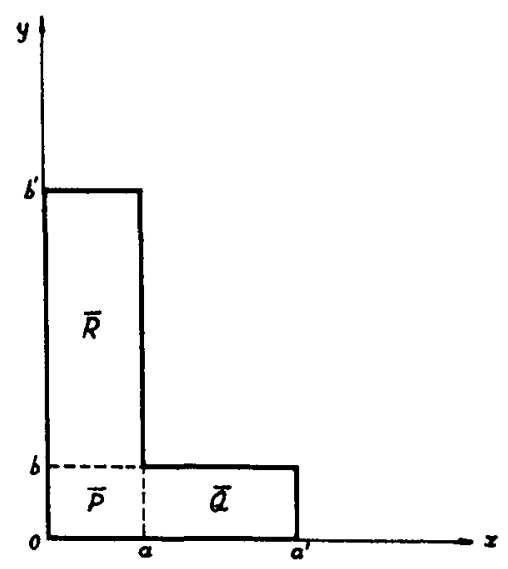

FIG. 1.

2. Basic equations. Our problem consists in determining a function $F(x, y)$ that satisfies the equation

where

$$
\nabla^{2} F=-2 \quad\left(\nabla^{2}=\frac{\partial^{2}}{\partial x^{2}}+\frac{\partial^{2}}{\partial y^{2}}\right)
$$

$$
F=0 \quad \text { on the boundary. }
$$

The torsional rigidity $D$ is given by

$$
D=2 \mu \iint_{S} F(x, y) d x d y
$$


where $\mu$ is the rigidity of the material and $S$ is the region of the cross-section.

The stress-components are given by

$$
\tau_{x z}=\alpha \mu \frac{\partial F}{\partial y}, \quad \tau_{y z}=-\alpha \mu \frac{\partial F}{\partial x},
$$

where $\alpha$ is the constant twist per unit length.

3. The stress function. Let us put

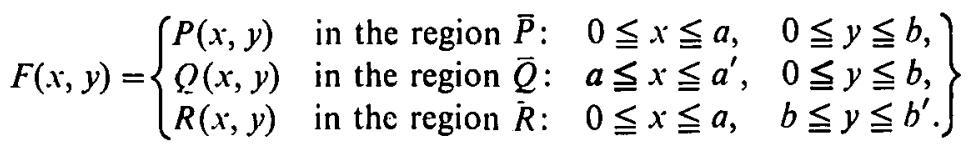

Equation (1) becomes

$$
\nabla^{2} P=-2, \quad \nabla^{2} Q=-2, \quad \nabla^{2} R=-2,
$$

and the boundary-condition (2) may be written

$$
\left.\begin{array}{l}
P(x, 0)=P(0, y)=0, \\
Q(x, 0)=Q(x, b)=Q\left(a^{\prime}, y\right)=0, \\
R(0, y)=R(a, y)=R\left(x, b^{\prime}\right)=0 .
\end{array}\right\}
$$

For continuity reasons it is necessary to have

$$
P(a, y)=Q(a, y), \quad P(x, b)=R(x, b)
$$

and

$$
\left[\frac{\partial P}{\partial x}\right]_{x=a}=\left[\frac{\partial Q}{\partial x}\right]_{x=a}, \quad\left[\frac{\partial P}{\partial y}\right]_{y=b}=\left[\frac{\partial R}{\partial y}\right]_{y=b} .
$$

Let us take

$$
\begin{aligned}
& P(x, y)=P_{1}(x, y)+P_{2}(x, y) \\
& Q(x, y)=Q_{1}(x, y)+Q_{2}(x, y) \\
& R(x, y)=R_{1}(x, y)+R_{2}(x, y)
\end{aligned}
$$

where $P_{1}(x, y), Q_{1}(x, y), R_{1}(x, y)$ are the stress-functions for the regions $\bar{P}, \bar{Q}, \bar{R}$, respectively, and $P_{2}(x, y), Q_{2}(x, y), R_{2}(x, y)$ are functions to be determined.

We have, consequently,

$$
P_{1}(x, y)=\sum_{m=1}^{\infty} \sum_{n=1}^{\infty} a_{m, n} \sin (m \pi x / a) \sin (n \pi y / b)
$$

where the dashes mean that only odd numbers $m$ and $n$ are summed over, and

$$
\begin{gathered}
a_{m, n}=\frac{32}{\pi^{4} m n\left(m^{2} a^{-2}+n^{2} b^{-2}\right)} \quad(m, n=1,3,5, \ldots), \\
Q_{1}(x, y)=y(b-y)-\frac{8 b^{2}}{\pi^{3}} \sum_{k=1}^{\infty} \frac{\operatorname{ch}\left\{k \pi\left(a^{\prime}+a-2 x\right) /(2 b)\right\} \sin (k \pi y / b)}{k^{3} \operatorname{ch}\left\{k \pi\left(a^{\prime}-a\right) /(2 b)\right\}},
\end{gathered}
$$




$$
R_{1}(x, y)=x(a-x)-\frac{8 a^{2}}{\pi^{3}} \sum_{k=1}^{\infty} \frac{\operatorname{ch}\left\{k \pi\left(b^{\prime}+b-2 y\right) /(2 a)\right\} \sin (k \pi x / a)}{k^{3} \operatorname{ch}\left\{k \pi\left(b^{\prime}-b\right) /(2 a)\right\}} .
$$

(The stress-function of a rectangle may be written in three forms. Here we have taken the stress-function in a different form for each region.)

For the functions $P_{2}(x, y), Q_{2}(x, y), R_{2}(x, y)$ we choose

$$
\begin{aligned}
& P_{2}(x, y)=\sum_{k=1}^{\infty}\left[A_{k} \sin (k \pi x / a) \operatorname{sh}(k \pi y / a)+B_{k} \sin (k \pi y / b) \operatorname{sh}(k \pi x / b)\right], \\
& Q_{2}(x, y)=\sum_{k=1}^{\infty} C_{k} \operatorname{sh}\left\{k \pi\left(a^{\prime}-x\right) / b\right\} \sin (k \pi y / b), \\
& R_{2}(x, y)=\sum_{k=1}^{\infty} D_{k} \operatorname{sh}\left\{k \pi\left(b^{\prime}-y\right) / a\right\} \sin (k \pi x / a),
\end{aligned}
$$

where $A_{k}, B_{k}, C_{k}, D_{k}(k=1,3,5, \ldots)$ are coefficients still to be determined.

Since the functions $P_{1}(x, y), Q_{1}(x, y), R_{1}(x, y)$ satisfy the equation $\nabla^{2} \psi=-2$, and since $P_{2}(x, y), Q_{2}(x, y), R_{2}(x, y)$ are harmonic functions, it follows that the functions $P(x, y), Q(x, y), R(x, y)$ given by (10), (11), (12), satisfy equations (6). It is easy to verify that the functions $P(x, y), Q(x, y), R(x, y)$ satisfy also the boundary conditions (7).

Conditions (8) are satisfied if

$$
C_{k} \operatorname{sh}\left\{k \pi\left(a^{\prime}-a\right) / b\right\}=B_{k} \operatorname{sh}(k \pi a / b), \quad D_{k} \operatorname{sh}\left\{k \pi\left(b^{\prime}-b\right) / a\right\}=A_{k} \operatorname{sh}(k \pi b / a) .
$$

Now, since

$\left[\frac{\partial P_{1}}{\partial x}\right]_{x=a}=-\frac{32 a}{\pi^{3}} \sum_{n=1}^{\infty} n^{-1} \sin (n \pi y / b) \sum_{m=1}^{\infty} \frac{1}{m^{2}+\left(a^{2} n^{2} / b^{2}\right)}=-\frac{8 b}{\pi^{2}} \sum_{n=1}^{\infty} n^{-2} \sin (n \pi y / b)$ th $(n \pi a /(2 b))$ and similarly,

$$
\left[\frac{\partial P_{1}}{\partial y}\right]_{y=b}=-\frac{8 a}{\pi^{2}} \sum_{m=1}^{\infty} m^{-2} \sin (m \pi x / a) \operatorname{th}(m \pi b /(2 a))
$$

conditions (9), after some computation, become

$$
\begin{gathered}
\sum_{\lambda=1}^{\infty}\left[\lambda B_{\lambda} \operatorname{ch} \frac{\lambda \pi a}{b}+\lambda C_{\lambda} \operatorname{ch} \frac{\lambda \pi\left(a^{\prime}-a\right)}{b}-\frac{8 b^{2}}{\lambda^{2} \pi^{3}} \text { th } \frac{\lambda \pi a}{2 b}-\frac{8 b^{2}}{\lambda^{2} \pi^{3}} \text { th } \frac{\lambda \pi\left(a^{\prime}-a\right)}{2 b}\right] \sin \frac{\lambda \pi y}{b} \\
=\frac{b}{a} \sum_{\lambda=1}^{\infty} \lambda A_{\lambda} \operatorname{sh} \frac{\lambda \pi y}{a},
\end{gathered}
$$

together with a similar equation in which $a$ and $b, a^{\prime}$ and $b^{\prime}, x$ and $y, A_{\lambda}$ and $B_{\lambda}$, and $C_{\lambda}$ and $D_{\lambda}$ are interchanged.

Multiplying (21) by $\sin (k \pi y / b)$ and integrating with respect to $y$ from 0 to $b$, we obtain

$$
\begin{gathered}
k B_{k} \operatorname{ch} \frac{k \pi a}{b}+k C_{k} \operatorname{ch} \frac{k \pi\left(a^{\prime}-a\right)}{b}-\frac{8 b^{2}}{k^{2} \pi^{3}} \text { th } \frac{k \pi a}{2 b}-\frac{8 b^{2}}{k^{2} \pi^{3}} \text { th } \frac{k \pi\left(a^{\prime}-a\right)}{2 b} \\
=\frac{2 a b k}{\pi} \sum_{\lambda=1}^{\infty} \frac{\lambda A_{\lambda}}{b^{2} \lambda^{2}+a^{2} k^{2}} \operatorname{sh} \frac{\lambda \pi b}{a} .
\end{gathered}
$$


A similar equation holds with the interchanges mentioned. Here we have used the fact that

$$
\int_{0}^{b} \operatorname{sh} \frac{\lambda \pi t}{a} \sin \frac{k \pi t}{b} d t=-\frac{a^{2} b k}{\pi\left(b^{2} \lambda^{2}+a^{2} k^{2}\right)} \operatorname{sh} \frac{\lambda \pi b}{a} \cos k \pi .
$$

Now, by (20), equation (21) becomes, after a direct calculation,

$$
\begin{aligned}
B_{k} \operatorname{sh} \frac{k \pi a^{\prime}}{b}=\frac{16 b^{2}}{k^{3} \pi^{3}} \frac{\operatorname{sh}\left\{k \pi\left(a^{\prime}-a\right) /(2 b)\right\}}{\operatorname{ch}\{k \pi a /(2 b)\}}\left\{k \pi a^{\prime} /(2 b)\right\} \\
+\frac{2 a b}{\pi} \operatorname{sh}\left\{k \pi\left(a^{\prime}-a\right) / b\right\} \sum_{\lambda=1}^{\prime} \frac{\lambda A_{\lambda} \operatorname{sh}(\lambda \pi b / a)}{b^{2} \lambda^{2}+a^{2} k^{2}}
\end{aligned}
$$

for $k=1,3,5, \ldots$. There is a similar equation for $A_{k}$. Put

$$
X_{\lambda}=\lambda A_{\lambda} \operatorname{sh}(\lambda \pi b / a), \quad Y_{\lambda}=\lambda B_{\lambda} \operatorname{sh}(\lambda \pi a / b) \quad(\lambda=1,3,5, \ldots) .
$$

We then get

$$
X_{k}=\alpha_{k}+\sum_{\lambda=1}^{\infty} \alpha_{k, \lambda} Y_{\lambda}, \quad Y_{k}=\beta_{k}+\sum_{i=1}^{\infty} \beta_{k, \lambda} X_{\lambda} \quad(k=1,3,5, \ldots),
$$

where, for odd positive integers $k$ and $\lambda$,

$$
\left.\begin{array}{l}
\alpha_{k}=\frac{16 a^{2}}{k^{2} \pi^{3}} \frac{\operatorname{sh}\left\{k \pi\left(b^{\prime}-b\right) /(2 a)\right\} \operatorname{sh}\{k \pi b /(2 a)\}}{\operatorname{ch}\left\{k \pi b^{\prime} /(2 a)\right\}}, \\
\beta_{k}=\frac{16 b^{2}}{k^{2} \pi^{3}} \frac{\left.\operatorname{sh}\left\{k \pi\left(a^{\prime}-a\right) /(2 b)\right\} \operatorname{sh}\{k \pi a / 2 b)\right\}}{\operatorname{ch}\left\{k \pi a^{\prime} /(2 b)\right\}},
\end{array}\right\}
$$

and

$$
\left.\begin{array}{l}
\alpha_{k, \lambda}=\frac{2 a b k \operatorname{sh}\left\{k \pi\left(b^{\prime}-b\right) / a\right\} \operatorname{sh}(k \pi b / a)}{\pi\left(a^{2} \lambda^{2}+b^{2} k^{2}\right) \operatorname{sh}\left(k \pi b^{\prime} / a\right)}, \\
\beta_{k, \lambda}=\frac{2 a b k \operatorname{sh}\left\{k \pi\left(a^{\prime}-a\right) / b\right\} \operatorname{sh}(k \pi a / b)}{\pi\left(b^{2} \lambda^{2}+a^{2} k^{2}\right) \operatorname{sh}\left(k \pi a^{\prime} \mid b\right)} .
\end{array}\right\}
$$

Write

$$
\left.\begin{array}{rl}
Z_{k} & =X_{k}, \quad c_{k}=\alpha_{k} \quad(k \text { odd }) ; \quad Z_{k}=Y_{k-1}, \quad c_{k}=\beta_{k-1} \quad(k \text { even }) ; \\
\gamma_{k, \lambda}=\alpha_{k, \lambda-1} \quad(k \text { odd } \lambda \text { even }), & \gamma_{k, \lambda}=0, \quad(k \text { odd, } \lambda \text { odd }) ; \\
\gamma_{k, \lambda}=\beta_{k-1, \lambda}, \quad(k \text { even, } \lambda \text { odd }), & \gamma_{k, \lambda}=0, \quad(k \text { even, } \lambda \text { even }) .
\end{array}\right\}
$$

Then the system (24) becomes

$$
Z_{k}=c_{k}+\sum_{\lambda=1}^{\infty} \gamma_{k, \lambda} Z_{\lambda} \quad(k=1,2,3, \ldots)
$$

We now show that this system is completely regular. If $k$ is odd, we have 


$$
\begin{aligned}
\sum_{\lambda=1}^{\infty}\left|\gamma_{k, \lambda}\right|=\sum_{\lambda=1}^{\infty} \alpha_{k, \lambda} & =\frac{2 a b k \operatorname{sh}\left\{k \pi\left(b^{\prime}-b\right) / a\right\} \operatorname{sh}(k \pi b / a)}{\pi \operatorname{sh}\left(k \pi b^{\prime} \mid a\right)} \sum_{\lambda=1}^{\infty} \frac{1}{a^{2} \lambda^{2}+b^{2} k^{2}} \\
& =\frac{\operatorname{sh}\left\{k \pi\left(b^{\prime}-b\right) / a\right\} \operatorname{sh}(k \pi b / a)}{2 \operatorname{sh}\left(k \pi b^{\prime} \mid a\right)} \text { th }\{k \pi b /(2 a)\} \leqq \frac{1}{4},
\end{aligned}
$$

since th $x \leqq 1$ and sh $x$ sh $y \leqq \frac{1}{2}$ sh $(x+y)$. If $k$ is even, we have, similarly,

$$
\sum_{\lambda=1}^{\infty}\left|\gamma_{k, \lambda}\right|=\sum_{\lambda=1}^{\infty} \beta_{k-1, \lambda} \leqq \frac{1}{4},
$$

It follows that the system (28) is completely regular.

We next consider the coefficients $c_{k}$ of the system. Since sh $x \operatorname{sh} y \leqq \frac{1}{2} \operatorname{ch}(x+y)$,

$$
0 \leqq c_{k} \leqq \frac{8 l^{2}}{\pi^{3}}, \text { where } \quad l=\max (a, b) .
$$

It is known [5] that in this case, the system (28) admits a unique bounded solution $0 \leqq Z_{k} \leqq K=32 l^{2} /\left(3 \pi^{3}\right)$, which may be approximated to by laborious computations, when the values of $a, b, a^{\prime}, b^{\prime}$ are given. The approximation can be carried out in several ways.

(i) One method is furnished by the following theorem: A completely regular system has always a unique and bounded solution, which can be found by the method of successive approximations, starting with any bounded system of initial values.

(ii) Another method is to find numerical sequences $Z_{k}^{\prime}$ and $Z_{k}^{\prime \prime}$ such that $Z_{k}^{\prime} \leqq Z_{k} \leqq Z_{k}^{\prime \prime}$, that is, to determine lower and upper approximations to $Z_{k}$. The values of $Z_{k}^{\prime}$ and $Z_{k}^{\prime \prime}(k=1$, $2, \ldots, N)$ are given by the finite systems of equations

$$
Z_{k}^{\prime}=c_{k}+\sum_{\lambda=1}^{N} \gamma_{k, \lambda} Z_{\lambda}^{\prime}, \quad Z_{k}^{\prime \prime}=c_{k}+K \sum_{\lambda=N+1}^{\infty} \gamma_{k, \lambda}+\sum_{\lambda=1}^{N} \gamma_{k, \lambda} Z_{\lambda}^{\prime \prime} .
$$

It can be shown that

$$
\lim _{N \rightarrow+\infty} Z_{k}^{\prime}=\lim _{N \rightarrow+\infty} Z_{k}^{\prime \prime}=Z_{k}
$$

For $k=N+1, N+2, \ldots$ we can take $Z_{k}^{\prime}=0, Z_{k}^{\prime \prime}=K$.

(iii) There exists a modified method of successive approximations. We write system (28) in the following form:

$$
-\sum_{\lambda=1}^{k-1} \gamma_{k, \lambda} Z_{\lambda}+\left(1-\gamma_{k, k}\right) Z_{k}=c_{k}+\sum_{\lambda=k+1}^{\infty} \gamma_{k, \lambda} Z_{\lambda} \quad(k=1,2, \ldots) .
$$

Taking the values of the unknown quantities, that appear on the right-hand side of (29), equal to zero and solving the recurrent system thus obtained, we find the first approximation for $Z_{k}$. Introducing these first approximations in the right-hand side of (29) and solving the new recurrent system thus obtained, we find the second approximations for $Z_{k}$, and so on. 
As soon as $Z_{k}$, and consequently $X_{k}$ and $Y_{k}$ are known, the stress-function can be obtained from (10), (11), (12), where $P_{1}(x, y), Q_{1}(x, y), R_{1}(x, y)$ are given by (13), (14), (15), (16). On taking into account (17), (18), (19), (20), (23), $P_{2}(x, y), Q_{2}(x, y), R_{2}(x, y)$ may be written as follows:

$$
\begin{aligned}
& P_{2}(x, y)=\sum_{k=1}^{\infty} \frac{1}{k}\left\{X_{k} \frac{\sin (k \pi x / a) \operatorname{sh}(k \pi y / a)}{\operatorname{sh}(k \pi b / a)}+Y_{k} \frac{\sin (k \pi y / b) \operatorname{sh}(k \pi x / b)}{\operatorname{sh}(k \pi a / b)}\right\}, \\
& Q_{2}(x, y)=\sum_{k=1}^{\infty} Y_{k} \frac{\operatorname{sh}\left\{k \pi\left(a^{\prime}-x\right) / b\right\} \sin (k \pi y / b)}{k \operatorname{sh}\left\{k \pi\left(a^{\prime}-a\right) / b\right\}}, \\
& R_{2}(x, y)=\sum_{k=1}^{\infty} X_{k} \frac{\operatorname{sh}\left\{k \pi\left(b^{\prime}-y\right) / a\right\} \sin (k \pi x / a)}{k \operatorname{sh}\left\{k \pi\left(b^{\prime}-b\right) / a\right\}} .
\end{aligned}
$$

4. The torsional rigidity. We shall denote by $\mu L(a, b)$, the torsional rigidity corresponding to a rectangular section with sides $a, b$. Here $L(a, b)$ is given by any of the formulae

$$
\begin{aligned}
& L(a, b)=\frac{256 a b}{\pi^{6}} \sum_{m=1}^{\infty} \sum_{n=1}^{\infty} \frac{1}{m^{2} n^{2}\left(m^{2} a^{-2}+n^{2} b^{-2}\right)}, \\
& L(a, b)=\frac{a^{3} b}{3}-\frac{64 a^{4}}{\pi^{5}} \sum_{m=1}^{\infty} \frac{1}{m^{5}} \text { th } \frac{m \pi b}{2 a}, \\
& L(a, b)=\frac{a b^{3}}{3}-\frac{64 b^{4}}{\pi^{5}} \sum_{m=1}^{\infty} \frac{1}{m^{5}} \text { th } \frac{m \pi a}{2 b},
\end{aligned}
$$

and may be calculated from tables.

For the rigidity of our beam we have

$$
D=2 \mu\left[\iint_{\bar{P}}\left(P_{1}+P_{2}\right) d x d y+\iint_{\bar{Q}}\left(Q_{1}+Q_{2}\right) d x d y+\iint_{\bar{R}}\left(R_{1}+R_{2}\right) d x d y\right] .
$$

Since $P_{1}(x, y), Q_{1}(x, y), R_{1}(x, y)$ are the stress-functions for the regions $\bar{P}, \bar{Q}, \bar{R}$, respectively, we have

$$
2 \iint_{\bar{P}} P_{1} d x d y=L(a, b), \quad 2 \iint_{\bar{Q}} Q_{1} d x d y=L\left(a^{\prime}-a, b\right), \quad 2 \iint_{\bar{R}} R_{1} d x d y=L\left(a, b^{\prime}-b\right) .
$$

As far as it concerns the other three integrals in (36), a simple calculation shows that

$$
\begin{aligned}
& \iint_{\bar{P}} P_{2} d x d y=\frac{2}{\pi^{2}} \sum_{k=1}^{\infty} \frac{1}{k^{3}}\left(a^{2} X_{k} \text { th } \frac{k \pi b}{2 a}+b^{2} Y_{k} \text { th } \frac{k \pi a}{2 b}\right), \\
& \iint_{\bar{Q}} Q_{2} d x d y=\frac{2 b^{2}}{\pi^{2}} \sum_{k=1}^{\infty} \frac{1}{k^{3}} Y_{k} \text { th } \frac{k \pi\left(a^{\prime}-a\right)}{2 b}, \\
& \iint_{\bar{R}} R_{2} d x d y=\frac{2 a^{2}}{\pi^{2}} \sum_{k=1}^{\infty} \frac{1}{k^{3}} X_{k} \text { th } \frac{k \pi\left(b^{\prime}-b\right)}{2 a} .
\end{aligned}
$$


Consequently, we have, from (36),

$D=D_{0}+\frac{4 \mu}{\pi^{2}} \sum_{k=1}^{\infty} \frac{1}{k^{3}}\left[a^{2} X_{k}\left(\operatorname{th} \frac{k \pi b}{2 a}+\right.\right.$ th $\left.\frac{k \pi\left(b^{\prime}-b\right)}{2 a}\right)+b^{2} Y_{k}\left(\right.$ th $\frac{k \pi a}{2 b}+$ th $\left.\left.\frac{k \pi\left(a^{\prime}-a\right)}{2 b}\right)\right]$,

where $D_{0}$ is the sum of the rigidities corresponding to the rectangular regions $\bar{P}, \bar{Q}, \bar{R}$; i.e.

$$
D_{0}=\mu\left[L(a, b)+L\left(a^{\prime}-a, b\right)+L\left(a, b^{\prime}-b\right)\right] \text {. }
$$

\section{REFERENCES}

1. K. Kötter, Úber die Torsion des Winkeleisens, S.-B. Kgl. Preuss. Akad. Wiss., Math.-Phys. Klasse (1908), 935-955.

2. E. Trefftz, UUber die Torsion prismatischer Stäbe von polygonalem Querschnitt, Math. Annalen 82 (1921), 97-112.

3. B. R. Seth, Torsion of beams, Proc. Cambridge Phil. Soc., 30 (1934), 392-403.

4. N. H. Arutyunyan, Solution of the problem of the torsion of a rod with a polygonal cross section, Prikl. Mat. Meh. 13 (1949), 107-112. 1958).

5. L. V. Kantorovich and V. I. Krylov, Approximate methods of higher analysis (Groningen,

\section{Institutul de Matematica}

BUCHAREST 3

RUMANIA 